\title{
Sequential analysis as a method for studying life courses: The Case of caregivers' well-being trajectories, Tiznit Morocco
}

\author{
Mohamed Elbouzidi ${ }^{1 *}$, and Mhamed Mahdane ${ }^{1}$ \\ 1 Research Laboratory in Humanities and Social Sciences, FLSH Agadir, Morocco
}

\begin{abstract}
Being a caregiver offers a sense of usefulness and satisfaction. Similarly, this caregiving function hurts all areas of the caregiver's life. In this vein, we believe that approaching this topic based on the sequential approach is of great importance.

Indeed, we will first present the life-course approach as a method for analysing longitudinal quantitative data while highlighting the different stages of sequence analysis as an appropriate analysis method. We will then discuss the importance of studying the life trajectories of caregivers as units of research in the life course approach.

The empirical demonstration of this paper originates from doctoral research in sociology on the life course of informal caregivers in the province of Tiznit in Morocco. Through this demonstration, we have crossed quantitative and qualitative analyses of life courses. This work will also show the relevance of sequence analysis and its adaptation to the life course approach of caregivers.
\end{abstract}

Keywords: sequence analysis, trajectories, caregiver, life-course approach.

\section{Introduction}

Contemporary society is undergoing an epidemiological and demographic transition, with all its consequences on the health of individuals and communities. We are currently witnessing an aging population and a remarkable increase in people with chronic diseases. Indeed, the notion of chronicity refers to the very long duration of the disease that can span the patient's lifetime. Beyond their period, chronic diseases are characterized, beyond their duration, by the extent of their impact on patient's daily lives and their families [1]. In this sense and according to the same author, chronic diseases affect different aspects of personal, emotional, family, social and professional life. Chronic diseases are characterized by their irreversibility, requiring a long term, sometimes for life. According to several authors, it was not only the patient's quality of life that was impacted but also the caregiver's quality of life was also degraded by the disease. Moreover, according to the French public health society (2009), chronic diseases impact the whole society and affect more and more people, families. According to Turcotte (2013), the consequences impact the caregiver, in particular on physical health, mental health, and on their participation in the labor market, as well as pressures on their finances; and reduced time available for other

\footnotetext{
Corresponding author: elbouzidi.mohamed9@gmail.com
} 
activities. Furthermore, according to the same author, employers and the state can also be affected, including absenteeism issues, decreased productivity, and reduced tax inflows [2]. The health status of the chronically ill person and the health status of the caregiver are interdependent. The caregiver is often in psychological distress, which increases the risk of suicide. Furthermore, the cessation of the caregiver's activity contributes to a decrease in their standard of living, knowing that social assistance will never cover all of the expenses caused by a chronic illness [3].

In our context, the population of caregivers continues to grow. However, we do not have figures illustrating the number of caregivers in Morocco. Given the number that will be remarkable in the following decades, in addition to the suffering of these collaborators of care which is often ignored. It seems essential to understand the reality of caregivers better. As described above, informal caregivers of chronically ill patients are, to this day, crucial actors in the health care system. They play an indispensable role in the care of patients. They contribute considerably through their emotional, moral, and material support to the therapeutic accompaniment of the person being cared for. They devote more time to the patients while carrying out the tasks and acts of daily life.

This study aims to propose a way of understanding the effect of chronic illness on life trajectories, an innovative method based on life course and sequential analysis.

This paper presents the sequential approach applied to the life course of informal caregivers of people with a chronic disease while highlighting its conceptual and empirical relevance.

First, we discuss the sequential approach and its importance for studying the life course of informal caregivers of chronically ill persons, particularly their perceived well-being trajectories. Then, in the methodology section, we explain the conceptualization of caregivers' trajectories, followed by an illustration of the main steps of the sequence analysis adopted in this study. Finally, in the results section, we discuss an empirical example of the sequential approach adapted to the life course of caregivers of chronically ill persons in the Moroccan province of Tiznit.

\subsection{The sequential approach as a framework for analysing the life course of informal caregivers of people with chronic illness:}

Approaching the issue of family caregivers of people with chronic illness under the aegis of a life course perspective finds its foundation in the possibility and ability of this approach to consider the different developmental dimensions of the individual. According to Carpentier $\&$ White (2013), the life course perspective attempts to organize the complexity of social life while considering the temporality, the socio-historical context, the structural constraints, and the agency of the social actors in an interdependent situation [4]. The life course as an individual biography allows for the analysis of individuals' lives by considering the socio-historical context in which these individuals evolve. According to Gaudet (2013), the institutional context and the individual trajectories of the life course thus take different directions [5]. Time and the individual are essential for the analysis of life courses or individual trajectories. Indeed, it is necessary to consider the temporal dimension and the "event" exposed to the person. In this sense, sequence analysis is the approach that allows us to analyze the individual's life, in particular, the life course of the caregiver.

This method had its origins in the Chicago School and was developed and improved by Abbott during the 1980s. This approach, used in biology to describe DNA sequences, is subsequently used in sociological and demographic studies. According to Mueller \& al. (2007), the purpose of the sequence analysis method is to obtain a typology of life courses. It will allow us to understand better the changes that may occur [6]. 
The literature is very diverse and shows a wide variety of statistical analysis techniques. Therefore, we limit ourselves to the important steps of this approach. So we can say that the analysis of the sequences takes place in four essential stages, namely: the Construction of the series, the Comparison of the sequences, the Creation of a typology, and finally, the Statistical analysis.

The first three steps, which are purely descriptive, make it possible to classify the trajectories according to a well-defined typology. It favors a classification of the courses according to typical classes. The last step is analytical.

\subsection{Optimal matching as a method of life-course analysis}

Optimal matching is one of the descriptive methods of sequence analysis. It makes it possible to identify regularities and similarities in the diversity of a corpus of sequences made up of a series of successive states and then construct typologies of "typical sequences"[7]. In the same sense, Optimal Matching Methods (OMM) considerably renews the quantitative perspectives of sociology and deserves to be integrated into the sociologist's toolbox to be used when the academic needs require it [8].

The optimal matching approach (OMA) has become the most popular technique for calculating distances between sequences since its introduction in the late 1980s in the social sciences by Abbott and Forrest (1986). The purpose of OMA is to build a typology of sequences, i.e. to match sequences of components. While it is impossible for the human eye to compare thousands of elements and how they are linked, OMA makes it possible to group them and identify ideal types [8].

The first step of this technique allows building a distance between sequences based on their comparison using three operations (insertion, deletion, or substitution of one element by another). This distance is established as the minimal cost to transform one sequence into another by these three operations. The cost of these three operations is indeed a parameter that gives excellent flexibility to these analyses [8]. The second step is the actual classification of the sequences, but other methods can also be used, such as Multidimensional Scaling.

\section{Methods}

To answer our problem and verify our hypotheses, we proceeded with a sequential analysis in two steps: first, a descriptive study by optimal matching that allows a typology of life trajectories, and second, identifying determinants that explain the life trajectories of informal caregivers. Then, we will analyze the different life trajectories according to the explanatory variables. These variables are the socio-demographic characteristics of the caregiver and the sociohistorical context. Thus, this approach allows us to study the life course as a function of time and the individual's sociohistorical context.

We forward for our study three groups of explanatory variables: a) socio-demographic characteristics of the caregiver (age, gender, education, residence, occupation, family status, number of children cared for), b) socio-demographic characteristics of the person being cared for (age, gender, occupation, family status), and c) characteristics and context of the assistance (dependence, need for assistance, the average duration of assistance, tasks provided). The different variables were tested one by one by the dispersion method based on the calculation of the Pseudo R2, to explain the life courses of informal caregivers of people with a chronic disease. Then, we selected only those variables that had a significant impact and explained the variability of the life trajectories. Finally, we used the decision trees or regression trees to explore the life courses of caregivers. According to Robette 
(2011), we can use a generalization of the principles of analysis of variance (ANOVA) and then an induction tree [9].

\subsection{The choice of the duration of observation of the sequences}

They are included in this part of the analysis the caregivers of people with chronic disease, falling within the province of Tiznit, aged 18 years or more, living with the patient and had to be involved in the care of patients during a period greater than or equal to two years. For reasons of dissimilarity of trajectories and to have sequences of the same length, we have opted for five years. Therefore, the constitution of the study population was aloud 181 caregivers of patients with chronic disease in Tiznit. They have observed annually 02 years before, the year of diagnosis, and 02 years after the illness (thus 05 successive observations), and taking as value for each observation one of the states defined for each trajectory.

\subsection{The choice of a dissimilarity measure and coding}

We have opted for the bottom-up classification of sequences. This method allows us to classify sequences according to their similarities, i.e. based on the comparison between two sequences while highlighting the degree of similarity of the states of each series. We have chosen a value of 1.5 for the cost of an insertion and deletion operation. And the substitution costs between states are determined from a cost matrix calculated based on the transition rates observed in our data. According to Studer (2014), this measure is an intermediate between the Hamming distance, which privileges timing or temporality, and the Levenshtein II distance, which permits order within trajectories [10].

The typology obtained through the sequence analysis (SA) corresponds to a nominal dependent variable. This can be analyzed by statistical methods such as regression models or multiple correspondence analyses, relating the biographies of caregivers to explanatory variables, such as socio-demographic characteristics (age, sex, place of residence, etc.). According to Studer (2012), we used dispersion analysis. In the same vein, dispersion analysis allows us to measure the variation explained by the variable and, therefore, the significance of the association between the life course and the explanatory variables. This analysis is based on the ANOVA method and provides a pseudo-R2.

\subsection{Data collection instrument:}

We conducted a retrospective longitudinal survey with a quantitative component based on the collection of life sequences over time and, more precisely, the situations experienced each year and five years. For the qualitative part, we reconstructed the life stories of the caregivers to extract the most significant trajectories, highlight the subjective data and complete the quantitative data.

The biographical grid or the "AGEVEN" form is the instrument of the data collection of our study.

\subsection{Ethical considerations}

We conducted this research following ethical considerations, including the rights to anonymity, confidentiality, and self-determination. We explained to participants that the results are for research purposes only and will be available for public dissemination and publication. We obtained written consent from almost all participants. 
For data processing, we used the statistical software R, version 4.0.2, with the package TraMineR.

\section{Results}

\subsection{The caregiver's typology of the well-being trajectories}

For almost all caregivers, we find a change in caregivers' well-being trajectory. And a transition to ill-being is observed with the illness' event of the persons being cared for. It suggests that chronic disease hurts the well-being of caregivers, especially for class $(1,2,3$, and 4). However, caregivers belonging to the other class (5 and 6) experience no change and no transition in their well-being trajectories. (Fig. 1)

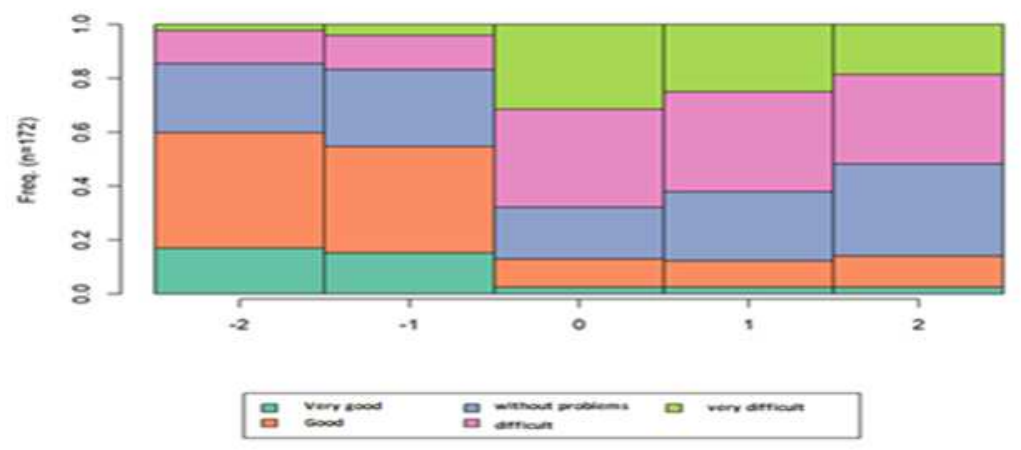

Fig. 1. The caregiver's typology of the well-being trajectories.

\subsection{Typology of caregivers' well-being trajectories by order of influence}

Several factors account for the variance in dissimilarity. Manly, caregivers' gender, education level. Also the duration of the assistance and the age and mental state of the person being cared for. To explain the typology of variability in the well-being trajectory, we opted for the induction tree classification.

In this sense, the most discriminating factor of the well-being trajectory typology is the duration of the care of the person being cared for. Caring for the person with the chronic disease for more than 24 years increases the chance of belonging to the "very difficult" well-being trajectory typology. For caregivers whose duration of care for the person with the chronic disease is less than 24 years, the most discriminating variable is the duration of care. Taking care of their sick relative for more than fifteen years is associated with the caregiver's level of education. Having a primary level of education or being illiterate favors the caregiver to belong to the typology of "very difficult" and "difficult" trajectory of wellbeing, especially after the chronic illness of the person being cared for. On the other hand, caregivers with high school education and higher belong to the "no problems" and "difficult" well-being trajectory typology after the chronic illness event. For caregivers with less than 15 years of caregiving experience, the most discriminating factor in the variability of the well-being trajectory is the person's age being cared for. The fact that the person being cared for is less than 20 years increases the probability of belonging to the "very difficult" and "difficult" well-being trajectory. Caregivers whose age of the person being 
cared for is less than or equal to 49 years favor belonging to the "no problems" wellness trajectory. (Fig. 2)
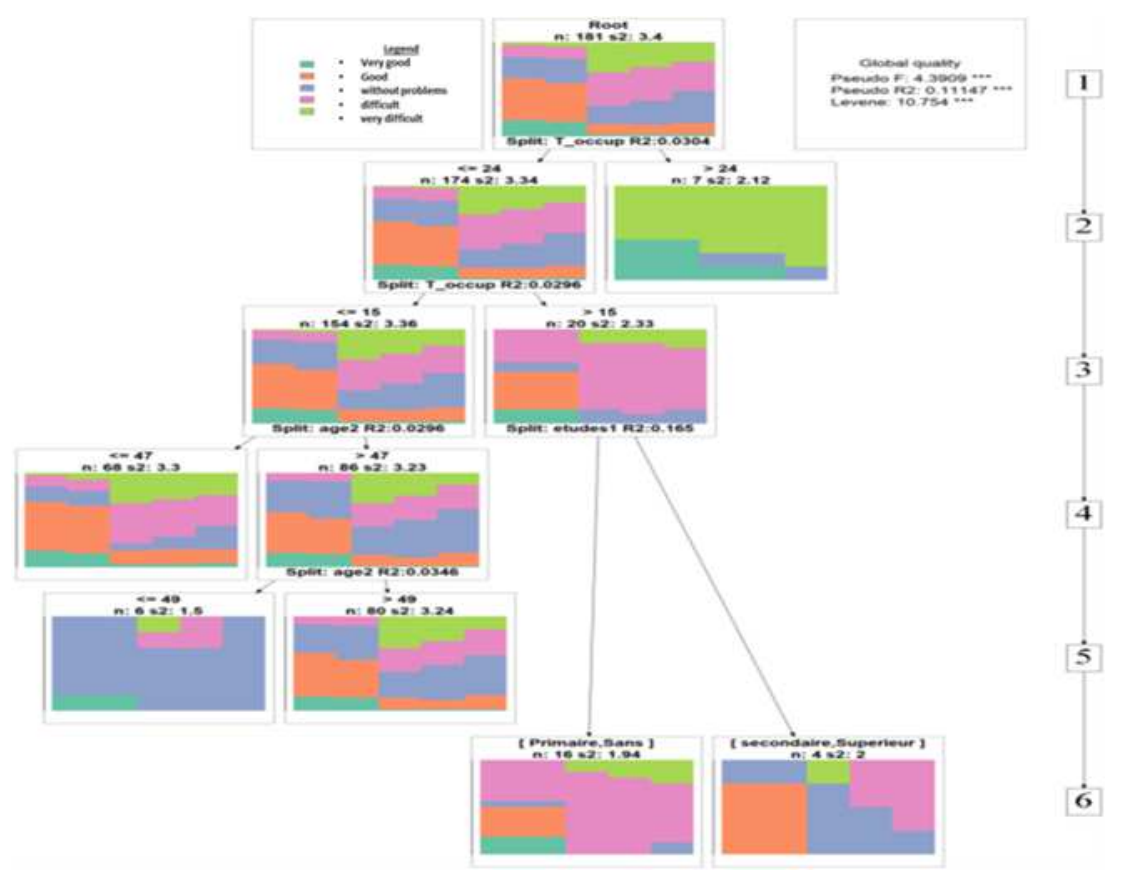

Fig. 2. the factors most associated with well-being trajectory by the induction tree.

\section{Discussion}

Indeed, the results obtained show that most informal caregivers presented a rupture in their well-being trajectories and that their health was affected. Based on the life stories of the caregivers, the analysis of feelings highlighted a set of emotional manifestations with the event of the illness of their loved ones. Although the caregivers showed positive feelings, most of the caregivers of people with chronic diseases showed various feelings, including anger, sadness, fear, disgust, and in general, some of the caregivers showed negative feelings. This is consistent with several studies, including the study by Thomas and Secall (2005), Bruegger (2011), and Ramelli (2016), who demonstrated the most frequently cited emotions by caregivers and their impact on life. In addition to these emotions, another feeling that has been manifested by caregivers of patients with chronic disease in Tiznit is exhaustion. What is so argued by the words of Verrecchia (2015), explaining that the state of exhaustion affects the physical, emotional, and intellectual side and which is the consequence of the stress felt by an individual placed in a situation from which he becomes unable to meet the demands of his profession [11]. The exhaustion felt by caregivers of patients with chronic disease may be closely related to the gender of the caregiver as women have more feelings of fatigue than male caregivers. Feelings of burnout may also be associated with the age of the caregiver. The nature of the assistance provided by the caregiver may influence the degree of caregiver burnout. Indeed, Bruegger (2011) demonstrated that it is thus easily understandable that caregivers are overworked, even depressed in the face of the workload imposed on them [12]. 
Similarly, the results of our research have highlighted that the variability of the typology of well-being trajectories is mainly associated with the gender of the caregiver, the caregiver's level of education, the age of the person being cared for, the duration of care, and the mental state of the person being cared for. From this point of view, the trajectory of wellbeing "very difficult years" or malaise is typical of the trajectories of informal female caregivers, especially with the event of the illness of the person being cared for. The typology of the well-being trajectory of male caregivers is characterized by "years without problems". However, as soon as the chronic illness of the person being cared for occurs, we find that the most dominant trajectory is "difficult years" or ill-being, with a regression over the years of the loved one's illness. Our findings are consistent with the study[13], illustrating that male partners feel the consequences of their partner's chronic illness less than female partners. Also, for women, their partner's chronic illness thus seems more disruptive than for men.

Regarding the level of education of the caregivers, we notice that the illiterate caregivers or those with a low level of education are characterized by a trajectory of well-being that evolves towards ill-being, especially after the event of the chronic illness of the person being cared for, witch consistent with the study [13] that illustrate that informal caregivers with higher levels of education were no less likely to feel physically burdened than with those with lower levels of education, but they were more likely to feel mentally burdened.

In this sense, the trajectory of well-being or health of the informal caregivers of people with chronic disease underwent remarkable biographical breaks, and that the evolution of this trajectory changed during the period of the study, which suggests that the chronic illness of the person being cared for influenced the well-being trajectory of the informal caregivers and caused a break in the health trajectory of the informal caregivers.

\section{Conclusion}

As one of the first studies on informal caregivers of people with chronic illness, this study highlighted the issue of informal caregivers, which has become increasingly topical, particularly in the context of demographic and epidemiological transition.

Indeed, we adopted the life course theory and, more precisely, the sequential approach. This approach is designed to analyze the life course and, the individual trajectories of caregivers by taking into consideration time and the individual. Just as this approach provides the researcher with data that can be processed and analyzed quantitatively through sequence analysis, the researcher also has another possibility to study the life history of the caregivers through the reconstruction of the data of the biographical grids "AGVEN" as life stories, thus allowing for qualitative processing and analysis of the collected data.

The biographical approach also offers an opportunity and flexibility to study globally all aspects of the life course and the different life trajectories as well as the socio-historical contexts. And the interaction between the individual's life spheres and its effect on the development of the individual in general. This is made possible by the processing of biographical data through multidimensional sequence analysis, which provides the social scientist with a robust tool for analyzing the links between different life trajectories while considering the principles of the life course.

In terms of contribution, our work was able to study the life course of informal caregivers innovatively. To our knowledge, it is the first time that the biographical approach and sequence analysis has been used in this sense. This approach has also allowed us to open the door to other research in the field of health sociology, in particular, the life course of informal caregivers and the life course of patients and their interactions. 


\section{References}

1. Yacine Thiam. Comprendre le vécu et les ressentis des patients chroniques à la suite d'un [pro- gramme] d'éducation thérapeutique en court séjour : le cas des patients cardiovasculaires du CHU Clermont-Ferrand. Médecine humaine et pathologie. Université d'Auvergne - Clermont-Ferrand I, 2012. Français. NNT : 2012CLF1MM14 . tel-01165179

2. M. Turcotte, "Être Aidant Familial : Quelles Sont Les Conséquences ?," Regard sur la société Can., vol. 75, p. 14, 2013, [Online]. Available: http://www.statcan.gc.ca/pub/75-006-x/2013001/article/11858-fra.pdf.

3. S. Santé publique, "Mieux vivre avec une maladie auquotidien chronique," 2009, [Online].

Available: https://www.sfsp.fr/images/docs/lire_et_ecrire/la_collection_sante_societe/sfsp_sante societe_15.pdf.

4. N. Carpentier and D. White, "Perspective des parcours de vie et sociologie de l'individuation," Vol. 45, Number 1, Spring 2013, 2013, doi: https://doi.org/10.7202/1016404ar.

5. P. de M. Gauthier and F. de Montigny, "Théorie du parcours de vie. cahier de recherche," Cah. Rech. 6, vol. Gatineau, p. 22, 2014.

6. N. S. Mueller, M. Studer, and Gilbert Ritschard, "Classification de parcours de vie `a l'aide de l'optimal matching," XIVe Rencontre la Société Francoph. Classif., no. Sfc 2007, pp. 157-160, 2007, [Online]. Available: http://archiveouverte.unige.ch/unige:4532 Disclaimer:

7. C. Nestrigue, L. Com-Ruelle, and D. Bricard, Analyse séquentielle et déterminants des parcours de soins en phase post-aiguë d'un Accident vasculaire cérébral (AVC). 2019.

8. L. Lesnard and T. de Saint Pol, "Introduction aux méthodes d'appariement optimal (optimal matching analysis)," Bull. Méthodologie Sociol., vol. 90, no. 1, pp. 5-25, 2006, doi: 10.1177/075910630609000103.

9. N. (Ined) Robette, "Explorer et décrire les parcours de vie: les typologies de trajectoires," pp. 1-54, 2011.

10. F. Rossignon, M. Studer, J.-A. Gauthier, and J.-M. Le Goff, "Sequence History Analysis (SHA): Estimating the Effect of Past Trajectories on an Upcoming Event," pp. 83-100, 2018, doi: 10.1007/978-3-319-95420-2_6.

11. S. Verrecchia, "le fardeau de la prise en charge par l'aidant naturel du patient atteint de maladie d'alzheimer (etude qualitative sur les facteurs favorisant l'épuisement)," l'Université Claude Bernard-Lyon 1, 2015.

12. S. Bruegger, "Les émotions et le bien être psychique des aidants naturels accompagnant des personnes atteintes de démence," Haute Ecole de Santé, Fribourg, 2011.

13. A. Goldberg et al., "Family impact and economic burden among caregivers of children with chronic kidney disease in Assiut, Egypt," Arch. Gerontol. Geriatr., vol. 19, no. 1, pp. 332-343, 2016, doi: 10.1016/j.archger.2009.04.006.

14. Oedekoven, M., Amin-Kotb, K., Gellert, P., Balke, K., Kuhlmey, A., \& Schnitzer, S. (2019). Associations between informal caregivers' burden and educational level. GeroPsych: The Journal of Gerontopsychology and Geriatric Psychiatry, 32(1), 1929. https://doi.org/10.1024/1662-9647/a000199 
15. "Taking Off Again" Copyright (C) 2020 The R Foundation for Statistical Computing Platform: x86_64-w64-mingw32/x64 (64-bit), (2020-06-22) -

16. Gabadinho, A., Ritschard, G., Müller, N. S., \& Studer, M. (2011). Analyzing and Visualizing State Sequences in $\mathrm{R}$ with TraMineR. Journal of Statistical Software, 40(4), 1-37. DOI http://dx.doi.org/10.18637/jss.v040.i04. 